\title{
Influência da intensidade da dor sobre as respostas nas escalas unidimensionais de mensuração da dor em uma população de idosos e de adultos jovens ${ }^{1}$
}

\section{Influence of pain intensity on the unidimensional scales responses of pain measurement in an elderly and young adults population}

\author{
Adriano Polican Ciena'; Rutineia Gatto ${ }^{3}$; Vanessa Cerqueria Pacini'; Vivian \\ Viani Picanço ${ }^{5}$ Ismaelino Mauro Nunes Magno ${ }^{6}$; Eduardo Alexandre Loth ${ }^{7}$
}

\section{Resumo}

A dor é definida como uma desagradável experiência sensorial e emocional associada a um dano atual ou potencial do tecido, ou descrita em termos deste dano, recebendo a denominação de " 5 sinal vital". Objetivou-se, neste estudo, avaliar a influência da intensidade da dor sobre as respostas nas escalas unidimensionais de mensuração de dor em idosos e adultos jovens internados no Hospital Universitário do Oeste do Paraná. Foi avaliada a dor de dois grupos de pacientes, idosos (GI) e adultos jovens (GII), contendo 100 indivíduos cada grupo, do período de abril a julho de 2006, que foram selecionados conforme critérios de inclusão e exclusão da pesquisa. A avaliação da dor foi realizada através das escalas: Escala Visual Analógica (EVA), Escala Visual Numérica (EVN) e Escala de Fáceis (EF). Os resultados apontaram forte nível de concordância entre as escalas no grupo GII, apresentando significância estatística $(\mathrm{p}<0,05)$ nas três classificações de dores, porém no grupo GI observou-se fraca concordância entre as escalas ( $<<0,05)$ da EVN na classificação da dor leve. Conclui-se que os resultados demonstraram a existência de influência da intensidade de dor sobre as respostas das escalas no grupo GI, porém no grupo GII não se observou influência da intensidade da dor sobre as respostas.

Palavras-chave: Dor. Medição da dor. Idoso.

\begin{abstract}
Pain is defined as an unpleasant sensorial and emotional experience associated to a current or potential damage to the tissue, or described in terms of this damage, as the " $5^{\text {th }}$ vital sign". The purpose of this study was to evaluate the influence of the pain intensity on the responses in unidimensional scales of pain measurement in elderly people and young adults who were admitted to the Hospital Universitário do Oeste do Paraná. The pain was evaluated in both groups, the elderly (GI) and young adults (GII),
\end{abstract}

1 Estudo realizado no Hospital Regional do Oeste do Paraná.

2 Graduado em Fisioterapia, pela Universidade Estadual de Londrina-UEL, Especialista em Fisioterapia Ortopédica e Traumatológica e em Fisioterapia Geriátrica pela Universidade Estadual do Oeste do Paraná - UNIOESTE campus Cascavel-PR e Docente do Curso de Biomedicina da Universidade Parananense - UNIPAR, campus Cascavel. E-mail: adrianociena@hotmail. com.

3 Especialista em Fisioterapia Geriátrica pela Universidade Estadual do Oeste do Paraná - UNIOESTE campus Cascavel-PR.

4 Acadêmica do Curso de Fisioterapia Universidade Estadual do Oeste do Paraná - UNIOESTE campus Cascavel-PR.

5 Acadêmica do Curso de Fisioterapia Universidade Estadual do Oeste do Paraná - UNIOESTE campus Cascavel-PR.

6 Graduado em Biomedicina, pela Universidade Federal do Pará-UFPA, Doutorando em Doenças Tropicais - UFPA e Coordenador do Curso de Biomedicina - UNIPAR/ campus Cascavel-Pr.

7 Fisioterapeuta, Mestre pelo Programa de Pós-graduação em Distúrbios da Comunicação Humana da UFSM /RS, docente e Coordenador do Curso de Fisioterapia da UNIOESTE - campus de Cascavel/PR. 
with a hundred individuals in each group, from April to July 2006, who were selected according to the research inclusion and exclusion criteria. Pain evaluation was carried out through the following scales: the Analogical Visual Scale (AVS), the Numerical Visual Scale (NVS) and the Easy Scale (ES). Results showed a strong level of concordance between the GII-group scales, presenting statistical significance $(p<0,05)$ of the NVS in mild pain classification. It is concluded that the results showed the existence of the influence of pain intensity on the responses of GI-group scales, which did not happen in the GII group.

Keywords: Pain. Pain measurement. The elderly.

\section{Introdução}

Segundo a International Association for the Study of Pain (IASP), a dor foi oficialmente definida em 1986 como sendo uma "desagradável experiência sensorial e emocional associada a um dano atual ou potencial do tecido ou descrita em termos deste dano" (MICELI, 2002). Em 1996, a American Pain Society (APS) a introduziu como "o $5^{\circ}$ sinal vital" (GALVÃO; SILVA, 2005; BERMUDEZ et al., 2003).

A dor sofre variações até na maneira como o indivíduo a relata, pois há relacionamento com inúmeros fatores pessoais tais como: sexo, idade, personalidade, herança étnica/cultural, necessidades comportamentais e experiências dolorosas pregressas (AUGUSTO et al., 2004). Assim, Pereira e Sousa (1988) relatam que, diante da subjetividade, complexidade e multidimensionalidade da experiência dolorosa, o primeiro desafio no combate à dor inicia-se na sua mensuração.

$\mathrm{Na}$ tentativa de documentar de forma objetiva a dor dos pacientes, foram desenvolvidos instrumentos unidimensionais e multidimensionais para sua mensuração. Os instrumentos unidimensionais são os mais utilizados, e quantificam apenas a severidade ou a intensidade da dor e, como exemplos desses instrumentos, têm-se as escalas numéricoverbais e analógico-visuais. Já os instrumentos multidimensionais são empregados para avaliar e mensurar as diferentes dimensões da dor, como a sensitivo-discriminativa e a afetivo-motivacional (PEREIRA; SOUSA, 1998; SOUSA, 2002).

Os instrumentos de avaliação de dor unidimensionais pela facilidade de aplicação, são os mais utilizados, sendo recomendada a utilização de mais de uma escala ou medida de avaliação de dor para que se possa perceber as vantagens e desvantagens de cada uma (ARAÚJO-SOARES; FIGUEIREDO, 2001).

Visto o exposto acima, objetivou-se, no presente estudo, avaliar a influência da intensidade da dor sobre as respostas nas escalas unidimensionais de mensuração de dor em idosos e adultos jovens internados no Hospital Universitário do Oeste do Paraná.

\section{Materiais e métodos}

O estudo quali-quantitativo, de corte transversal, foi previamente aprovado pelo Comitê de Ética em Pesquisa da Unioeste, campus de Cascavel, e registrado com o número 017611/2006.

A amostra foi composta por 200 pacientes internados no Hospital Universitário do Oeste do Paraná, no período de abril a julho de 2006, selecionados conforme critérios de inclusão e exclusão da pesquisa, e isso caracterizou a amostra como estratificada de acordo com a faixa etária e selecionada aleatoriamente.

Os pacientes foram divididos em dois grupos de 100 pacientes. O grupo I (GI) foi composto por indivíduos idosos (pessoas acima de 60 anos conforme o Ministério de Saúde do Idoso, por meio da Portaria $n^{\circ}$. 1.395/GM da Política Nacional de Saúde do Idoso (BRASIL, 1999), de ambos os sexos (59 homens e 41 mulheres com média de idade 65 \pm 7,51 anos) e idade máxima de 90 anos. O grupo II (GII) foi composto por indivíduos adultos jovens 
de ambos os sexos (60 homens e 40 mulheres), com idade entre 20 a 40 anos (média de idade de $32,5 \pm$ 5,84 anos).

O critério de inclusão respeitado para o estudo foi assinar de livre arbítrio o Termo de Consentimento Livre e Esclarecido e relatar a sensação de dor no ato da pesquisa.

Foram excluídos do estudo os pacientes com nível de consciência de Glasgow inferior a 13 pontos, voluntários dementes ou com outros distúrbios psiquiátricos, bem como os pacientes nãoenquadrados nas faixas etárias pré-determinadas. A escala de coma de Glasgow (ECGL) é um instrumento de avaliação neurológico utilizado em pacientes apresentando comprometimento do Sistema Nervoso Central ou fatores que atenuem o nível de consciência, classificação conforme pontuação em categorias: grave (3 a 8 pontos), moderado( 9 a 12 pontos) e leve (13 a 15 pontos) (KOIZUMI; ARAÚJO, 2005).

Foi utilizado um questionário com o objetivo de angariar os dados pessoais, inclusive o nível de escolaridade, nível de Glasgow. Para correta avaliação da intensidade da dor foi necessário uma linguagem comum entre o avaliador devidamente treinado e os voluntários, traduzindo na padronização e ensinamento das escalas, assegurando aos voluntários a compreensão adequada e o seu significado. Em seguida, foi realizada a avaliação da dor, indicado pelos voluntários, através das seguintes escalas unidimensionais: Escala de Dor Visual Analógica (EVA) (SOUZA, 2002), Escala de Dor Visual Numérica (EVN) (PEREIRA; SOUSA, 1998), Escala de Dor de Faces (EF) (PEREIRA; SOUSA, 1998), com 6 faces.

A EVA consiste em uma linha horizontal com 10 centímetros de comprimento, assinalada em uma de suas extremidades a classificação "SEM DOR" e, na outra, a classificação "DOR MÁXIMA". O voluntário realiza a marcação com um traço no ponto que representa a intensidade de sua dor. A EVN consiste em uma régua dividida em onze partes iguais, numeradas sucessivamente de 0 a 10 . O voluntário refere à equivalência entre a intensidade da sua dor e uma classificação numérica, sendo que 0 corresponde a classificação "SEM DOR" e a 10 "MÁXIMA DOR". Na EF o voluntário indica a intensidade de sua dor de acordo com a mímica representada em cada face desenhada, sendo que à expressão de felicidade corresponde a classificação "SEM DOR" e à expressão de máxima tristeza corresponde à classificação "DOR MÁXIMA".

A análise estatística foi realizada por meio da estatística descritiva, com a utilização do programa Excel 2003/XP da Microsoft Oficce ${ }^{\circledR}$, no qual as variáveis qualitativas foram apresentadas por meio de tabelas de freqüências absolutas enquanto as variáveis quantitativas foram analisadas através de médias, desvios-padrão, e valores máximo (Máx) e mínimo (Mín).

A estatística analítica foi realizada com a utilização do Coeficiente de Concordância de Kappa, comparando-se as respostas das escalas EVA e EVN, que foram divididas em 3 níveis, de 0 a 3 foi considerado "dor leve", 4 a 7 "dor moderada" e acima de 8 pontos, "dor intensa". Para a EF foi admitido "dor leve" equivalente a face 2, "dor moderada" para as faces 3 e 4 e "dor intensa" para as faces 5 ou 6 . O índice que mensura o grau de concordância de Kappa utilizado foi o mesmo adotado por Farias Junior, Pires e Lopes (2002). A EVA foi admitida como variável independente do estudo, para tanto foi utilizado o Software SPSS ${ }^{\circledR}$ versão 13.

\section{Resultados}

No que tange ao perfil educacional dos indivíduos avaliados no estudo, o GI apresentou nível de escolaridade baixo comparado ao GII, com indivíduos desde analfabetos até o $2^{\circ}$ grau incompleto, já o GII apresentou melhor distribuição quanto ao nível de escolaridade, porém, em ambos os grupos, verificou-se elevado número de indivíduos com $1^{\circ}$ grau incompleto (Tabela 1$)$. 
Tabela 1. Apresentação da distribuição de acordo com o perfil educacional dos indivíduos do GI e GII.

\begin{tabular}{ccc}
\hline Nível de escolaridade & Número de Indivíduos & \\
\hline & GI & GII \\
$3^{\circ}$ grau completo & - & 3 \\
$3^{\circ}$ grau incompleto & - & 1 \\
$2^{\circ}$ grau completo & - & 26 \\
$2^{\circ}$ grau incompleto & 2 & 9 \\
$1^{\circ}$ grau completo & 9 & 18 \\
$1^{\circ}$ grau incompleto & 56 & 42 \\
Analfabeto & 33 & 1 \\
Total & $100 \%$ & $100 \%$ \\
\hline
\end{tabular}

Fonte: Autores.

Em relação às escalas de avaliação da dor, o GI apresentou média de $3,70 \pm 2,29$ pontos na EVA, (9,8-0,3 pontos). Na EVN os resultados apontaram a média de $5 \pm 2,34$ pontos (1-10 pontos). Na EF, o escore médio obtido foi de $4 \pm 1,75$ pontos (3-10 pontos).
O GII apresentou média de $3,70 \pm 2,26$ pontos na EVA(0,7-10 pontos), 4,5 $\pm 2,3$ pontos (1-10 pontos) na EVN, na EF $4 \pm 1,80$ pontos (2-10 pontos) (Tabela 2).

Tabela 2. Apresentação das medidas de tendência central e medidas de dispersão em pontos, dos resultados obtidos através da aplicação das escalas de dor, em ambos os grupos.

\begin{tabular}{lllll}
\hline & Escalas & \multicolumn{1}{r}{ Média } & Máx & \multicolumn{1}{c}{ Mín } \\
\hline \multirow{3}{*}{ GI } & EVA & $3,70 \pm 2,29$ & 9,8 & 0,3 \\
& EVN & $5 \pm 2,34$ & 10 & 1 \\
& EF & $4 \pm 1,75$ & 10 & 3 \\
& & & 10 & 0,7 \\
\multirow{2}{*}{ GII } & EVA & $3,70 \pm 2,26$ & 10 & 1 \\
& EVN & $4,5 \pm 2,3$ & 10 & 2 \\
\hline
\end{tabular}

Nota: Máx = valor máximo, Mín = valor mínimo.

Fonte: Autores.

Quanto à classificação da dor no grupo GI, na EVA, 32\% dos idosos foram classificados como portadores de dor leve, $58 \%$ de dor moderada, e $10 \%$ de dor intensa. Na EVN, $22 \%$ foram classificados como portadores de dor leve, $58 \%$ de dor moderada, e $20 \%$ de dor intensa. Já na EF, $80 \%$ foram classificados como de dor leve, $17 \%$ de dor moderada e $3 \%$ de dor intensa.
No grupo GII, 40\% dos jovens adultos foram classificados como portadores de dor leve pela EVA, $52 \%$ dor moderada e $8 \%$ dor intensa. Na EVN 36\% foram classificados como portadores de dor leve, $49 \%$ de dor moderada, e $15 \%$ de dor intensa. Porém, na EF $82 \%$ foram classificados com dor leve, $13 \%$ com dor moderada, e 5\% com dor intensa. 
Tabela 3. Apresentação do Coeficiente Kappa das escalas de avaliação de dor com EVA observados em GI e GII, nas 3 classificações de intensidade de dor.

\begin{tabular}{cccc}
\hline Grupo & Classifcação da dor & EVN & EF \\
\hline \multirow{2}{*}{ GI } & Dor leve & $\mathrm{K}=0.37^{*}$ & $\mathrm{~K}=0.27$ \\
& Dor moderada & $\mathrm{K}=0.22$ & $\mathrm{~K}=0.06$ \\
& Dor intensa & $\mathrm{K}=0.25$ & $\mathrm{~K}=0.15$ \\
& Dor leve & $\mathrm{K}=0.68^{*}$ & $\mathrm{~K}=0.53^{*}$ \\
& Dor moderada & $\mathrm{K}=0.62^{*}$ & $\mathrm{~K}=0.46^{*}$ \\
& Dor intensa & $\mathrm{K}=0.68^{*}$ & $\mathrm{~K}=0.63^{*}$ \\
\hline
\end{tabular}

$* \mathrm{p}<0,05$

A Tabela 3 mostra o grau de concordância por meio do Índice de Kappa entre as classes de dor dos grupos. Observa-se que no grupo GII houve moderado grau de associação entre as respostas da EVA com a EF nas duas primeiras classificações e concordância forte, na classificação dor intensa. Contudo, com a EVN, os índices de concordância observados foram fortes.

No grupo GI, observou-se forte associação da EVA com a EVN, porém os demais índices de associação apresentados entre as escalas foram fracos.

\section{Discussão}

A dor é considerada um sinal vital tão importante quanto os outros (freqüências cardíaca e respiratória, temperatura corpórea, pressão arterial) e deve sempre ser avaliada num ambiente clínico, para se empreender um tratamento ou conduta terapêutica. A eficácia do tratamento e o seu seguimento dependem de uma avaliação e mensuração da dor confiável e válida (PEDROSO; CELISH, 2006).

Nesse contexto, o presente estudo procurou avaliar a influência da intensidade da dor sobre as respostas nas escalas unidimensionais de mensuração de dor, aplicando-as em uma população de idosos e de adultos jovens, considerando a EVA, o referencial comparativo, dividindo as reposta das escalas em 3 classes.
O grau de concordância das respostas das escalas de dor observado no grupo de adulto jovens, classificado nos 2 primeiros níveis, apresentou índice de Kappa moderado, porém, com a EVN, observou-se forte índice de associação nos 3 níveis de dor. Cabe ressaltar que, neste grupo, todos os índices de concordância apresentaram significância estatística. No GI, o índice de concordância foi fraco, com significância estatística na classificação de intensidade dor leve e para as demais classificações no mesmo grupo, não apresentaram valores com significância estatística.

O presente estudo concorda com os resultados descritos por Santos et al. (2006), segundo o qual os idosos podem apresentar dor em dimensões aumentadas, motivo pelo qual os autores afirmam que a dor no idoso é difícil de ser relatada. Contudo, não foram encontrados na literatura compulsada estudos similares que propiciassem uma discussão mais aprofundada do tema deste trabalho.

Galvão e Silva (2005) mencionam que as escalas de avaliação da dor não se têm mostrado adequadas à população idosa. Os achados deste estudo corroboram com os autores acima, uma vez que se verificou que o índice de concordância das escalas com a EVA, nos idosos, demonstraram maior percentagem de respostas divergentes, sobretudo nos níveis de dor, aqui considerados moderados e fortes. 
No entanto, houve outro aspecto que se revelou determinante para o correto preenchimento dos instrumentos utilizados nesta pesquisa, e influenciou as respostas dos idosos, foi o nível cognitivo aliado à baixa escolaridade. De modo similar, Fávero e Soares (2002) relatam que o desenvolvimento cognitivo contempla fatores educacionais, sociais, culturais, lingüísticos e neurológicos. Seguindo a mesma linha, Charchat-Fichman et al. (2005), corroboram que o declínio cognitivo fisiológico normal que afeta os idosos.

A EVA é classificada como unidimensional, pois avalia somente uma das dimensões da experiência dolorosa, a intensidade (LIMA NETO; GOLDENBERG; JUCÁ, 2003). Jensen, Karroly e Braver (1986) citam que, para indivíduos jovens, esta escala apresenta alto índice de validade e confiabilidade, no entanto o aumento da idade está relacionado ao aumento de respostas incorretas para a EVA. Os dados desta pesquisa corroboram os achados dos autores citados, uma vez que os índices de concordância de Kappa das escalas com a EVA, no grupo de idosos, foram notoriamente menores.

Deve-se salientar que a concordância da EVN com a EVA apresentou índices maiores em ambos os grupos. Esse fato foi confirmado pelos escritos de Pedroso e Celish (2006), conforme os quais essas escalas têm a vantagem de facilitar o entendimento do indivíduo avaliado e, logo, facilitar a escolha da resposta adequada.

Assim, sugere-se que, para a avaliação da dor, deve ser considerada a faixa etária e o nível de escolaridade dos indivíduos. Para a avaliação em idosos, é importante considerar a diminuição fisiológica do nível cognitivo desse grupo, e optar pela utilização de outras formas de verificação e dimensionamento da dor.

Nesse sentido, e como alternativas às escalas utilizadas neste estudo, Chaves (2004) apresenta os seguintes itens como sinais de dor: relato verbal ou codificado, gestos protetores, expressão facial, distúrbio do sono, respostas autonômicas, comportamento expressivo, mudanças na alimentação, aparência abatida e interação reduzida com o ambiente.

\section{Conclusão}

Conclui-se que a intensidade da dor pode ter influenciado no índice de concordância das respostas da Escala Visual Analógica com as outras escalas utilizadas neste estudo, no grupo de idosos. Porém fatores como o nível cognitivo e grau de escolaridade não podem ser descartados. No grupo de adultos jovens, observou-se forte concordância entres as respostas das escalas, sugerindo que não houve influência da intensidade de dor sobre as respostas.

\section{Referências}

ARAÚJO-SOARES, V.; FIGUEIREDO, M. Dor: a hidra de sete cabeças. Revista Portuguesa de Psicossomática, Porto, v. 3, n. 2, p. 159-84, 2001.

AUGUSTO, A. C. C.; SOARES, A. P. S.; RESENDE, M. A.; PEREIRA, L. S. M. Avaliação da dor em idosos com doença de Alzheimer: uma revisão bibliográfica. Textos sobre envelhecimento, Rio de Janeiro, v. 7, n. 1, p. 1-10, 2004.

BERMUDEZ, C. C.; SOUZA, R. A.; SOUZA, C. M. F.; DIAS, E. M.; CASTRO, C. E. S. Impacto da dor na vida de portadores de disfunção temporomandibular. Journal of Applied Behavioral Science, Greenwich, v. 11, n. 2, p. 138-43, 2003.

BRASIL. Portaria no ${ }^{\circ}$ 1.395/GM, de 10 de Dezembro de 1999. Aprova a Política Nacional de Saúde do Idoso. Diário Oficial [da] República Federativa do Brasil, 13 dez. 1999, nº 237-E, p. 20, Seção 1.

CHARCHAT-FICHMAN, H.; CARAMELLI, P.; SAMESHIMA, K.; NITRINI, R. Declínio da capacidade cognitiva durante o envelhecimento. Revista Brasileira de Psiquiatria, São Paulo, v. 27, n. 12, p. 79-82, 2005.

CHAVES, L. D. Dor pós-operatória: aspectos clínicos e assistência de enfermagem. In: CHAVES, L. D.; LEÃO, E. R. Dor: $5^{\circ}$ sinal vital: reflexões e intervenções de enfermagem. Curitiba: Maio, 2004. p. 151-68.

FARIAS JUNIOR, J. C.; PIRES, M. C.; LOPES, O. S. Reprodutibilidade de um questionário para o levantamento 
de informações sobre comportamentos relacionados à saúde em adolescentes. Revista Brasileira de Ciência e Movimento, Brasília, v. 10, n. 3, p. 43-8, 2002.

FÁVERO, M. H.; SOARES, M. T. Iniciação escolar e a notação numérica: uma questão para o estudo do desenvolvimento adulto. Psicologia: Teoria e Pesquisa, Brasília, v. 18, n. 1, p. 43-50, 2002.

GALVÃO, O.; SILVA, M. G. Validade e fidedignidade preliminares da EVA modificada para a população idosa. EssFisionline, Setúbal, v. 1, n. 4, p. 22-30, 2005.

JENSEN, M. P.; KARROLY, P.; BRAVER, S. The measurement of clinical pain intensity: a comparison of six methods. Pain, Amsterdam, v. 27, n. 1, p. 117-26, 1986.

KOIZUMI, M. S.; ARAÚJO, G. L. Escala de Coma de Glasgow - subestimação em pacientes com respostas verbais impedidas. Acta Paulista de Enfermagem, São Paulo, v. 18, n. 21, p. 136-42, 2005.

LIMA NETO, E. V.; GOLDENBERG, A.; JUCÁ, M. J. Resultados imediatos da herniorrafia inguinal com anestesia local associada com sedação. Acta Cirúrgica Brasileira, São Paulo, v. 18, n. 5, p. 478-84, 2003.

MICELI, A. V. P. Dor crônica e subjetividade. Revista Brasileira de Cancerologia, Rio de Janeiro, v. 48, n. 3, p. 363-73, 2002.

PEDROSO, R. A.; CELISH, K. L. S. Dor: quinto sinal vital, um desafio para o cuidar em enfermagem. Texto \& Contexto Enfermagem, Florianópolis, v. 15, n. 2, p. 270-6, 2006.

PEREIRA, L. V.; SOUSA, F. A. E. F. Mensuração e avaliação da dor pós-operatória: uma breve revisão. Revista Latino-Americana de Enfermagem, Ribeirão Preto, v. 6, n. 3, p. 77-84, 1998.

SANTOS, C. C.; PEREIRA, L. S. M.; RESENDE, M. A.; MAGNO, F.; AGUIAR, V. Aplicação da versão brasileira do questionário de dor Mcgill em idosos com dor crônica. Acta Fisiátrica, São Paulo, v. 13, n. 2, p. 75-82, 2006.

SOUSA, F. A. E. F. Dor: o quinto sinal vital. Revista Latino-Americana de Enfermagem, Ribeirão Preto, v.10, n. 3, p. 446-7, 2002. 


\section{APÊNDICE A}

Instrumento de Avaliação

Nome:

Idade:

Grupo de inserção:

Grupo I ( ) Grupo II ( )

Sexo:

Feminino ( ) Masculino ( )

Escolaridade:

$1^{\circ}$ grau incompleto ( )

$1^{\circ}$ grau completo ( )

$2^{\circ}$ grau incompleto ( )

$2^{\circ}$ grau completo ( )

$3^{\mathrm{o}}$ grau incompleto ( )

$3^{\circ}$ completo ( )

Analfabeto ( )

Nível de Glasgow:

pontos

Doenças psiquiátricas existentes descritas no prontuário do pacientes:
$\operatorname{Sim}($ )
Não ( )

Obs: 


\section{APÊNDICE B}

Termo de Consentimento Livre e Esclarecido

Título do Projeto: Avaliação da Confiança das Escalas de Mensuração de Dor em uma População de Idosos e de Adultos

Pesquisador Responsável: Prof ${ }^{\circ}$. Eduardo Alexandre Loth

\section{Este projeto tem o objetivo de:}

Mensurar a dor de pacientes idosos e adultos, através de 3 escalas de avaliação e verificar se há correspondência entre as escalas, desenvolvendo a validade e fidedignidade das mesmas.

\section{E tem como justificativa:}

Utilizar inúmeras escalas de avaliação de dor, em populações de idosos e adultos internados no Hospital Universitário do Oeste do Paraná, possibilitando ao término do estudo, saber se há correspondência entre as escalas nestes indivíduos.

Para tanto será necessário realizar os seguintes procedimentos:

Os voluntários serão submetidos a avaliação da intensidade da dor, através das escalas: Escala de Dor Visual Analógica, Escala de Dor Visual Numérica, Escala de Dor de Faces. A Escala de Dor Visual Analógica consiste em uma linha horizontal com 10 centímetros de comprimento, que tem assinalada numa extremidade a classificação "Sem Dor" e, na outra, a classificação "Dor Máxima”. O voluntário terá que fazer um traço no ponto que representa a intensidade da sua Dor.

A Escala Visual Numérica consiste numa régua dividida em onze partes iguais, numeradas sucessivamente de 0 a 10 . O voluntário deverá fazer a equivalência entre a intensidade da sua Dor e uma classificação numérica, sendo que o 0 corresponde a classificação "Sem Dor" e a 10 a classificação "Dor Máxima”.

Na Escala de Dor de Faces será solicitado ao voluntário que classifique a intensidade da sua Dor de acordo com a mímica representada em cada face desenhada, sendo que à expressão de felicidade corresponde a classificação "Sem Dor" e à expressão de máxima tristeza corresponde a classificação "Dor Máxima". 


\section{Durante execução do projeto, não existe nenhum risco ou possibilidade de agravo à saúde.}

Após ler e receber explicações sobre a pesquisa, e ter meus direitos de:

1-Receber resposta a qualquer pergunta e esclarecimento sobre os procedimentos, riscos, benefícios e outros relacionados à pesquisa;

2-Retirar o consentimento a qualquer momento e deixar de participar do estudo;

3- Não ser identificado e ser mantido o caráter confidencial das informações relacionadas à privacidade.

4- Procurar esclarecimentos com o Comitê de Ética em Pesquisa da UNIOESTE, através do telefone 32203272, em caso de dúvidas ou notificação de acontecimentos não previstos.

Nome do sujeito:

Assinatura:

Eu, Eduardo Alexandre Loth, declaro que forneci todas as informações referentes ao estudo ao participante.

Assinatura do pesquisador

Data:

Telefone : $3220-3158$ 


\section{APÊNDICE C}

Escalas de Dor

Figura 1: Escala de Dor Visual Analógica

Sem dor

Dor máxima

Figura 2: Escala de Dor Visual Numérica

\begin{tabular}{|l|l|l|l|l|l|l|l|l|l|l|}
\hline 0 & 1 & 2 & 3 & 4 & 5 & 6 & 7 & 8 & 9 & 10 \\
\hline
\end{tabular}

Sem dor

Dor máxima

Figura 03: Escala de Dor de Faces

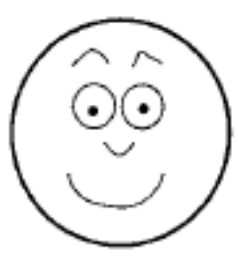

1

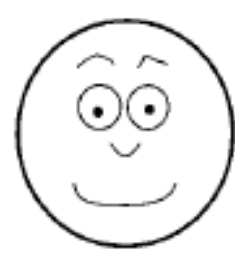

2

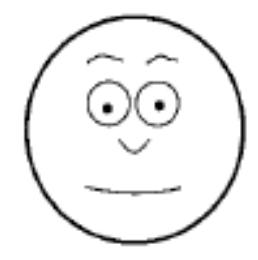

3

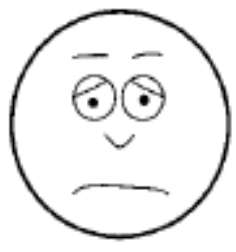

4

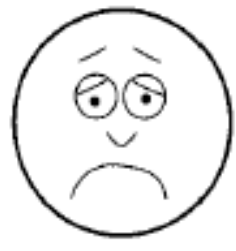

5

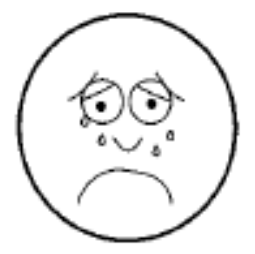

6 
